\title{
Optimizing the treatment of BRAF mutant melanoma
}

\author{
Jeff Settleman
}

Abstract
Selective inhibitors of the kinases BRAF and MEK for
the treatment of patients with otherwise refractory
BRAF mutant melanoma have demonstrated
impressive efficacy, and combination treatment with
these agents may prove to be even more effective.
However, these drugs are not curative, mainly because
of the relatively rapid development of drug resistance.
Furthermore, they can produce undesired, and even
unanticipated, side effects, including the emergence
of neoplastic lesions harboring activating RAS
mutations. Two recent reports reveal new
considerations for the optimal approach to targeting
this key oncogenic pathway in melanoma,
highlighting the importance of combination
treatment and therapeutic scheduling.

\section{Challenges in targeting the BRAF pathway}

The discovery of a highly recurrent mutant allele of the gene encoding the RAF-family kinase BRAF in human melanoma through cancer genome resequencing has set the stage for revolutionary new treatment strategies for this challenging disease [1]. This BRAF mutation, V600E, which has been detected in 50 to $60 \%$ of human melanomas, results in activation of the kinase; this drives the downstream MEK-ERK kinase signaling cascade, leading to proliferation and survival of cancer cells. Selective targeting of the BRAF or MEK kinases using targeted small-molecule inhibitory drugs in patients with BRAF V600E mutant melanoma has been associated with impressive clinical responses in more than half of treated patients - despite the presence of systemic disease $[2,3]$. However, as with other kinase-targeted anticancer drugs, the clinical benefit is typically short-lived due to the emergence of drug resistance, through a

Correspondence: Settleman.jeffrey@gene.com

Discovery Oncology, Genentech, 1 DNA Way, South San Francisco, CA 94080, USA

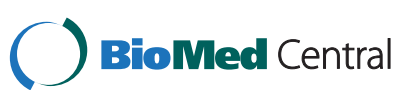

variety of molecular mechanisms [4]. Such findings have prompted substantial efforts to elucidate these mechanisms and to use that information to guide the discovery of second-generation drugs, drug combination strategies, and novel treatment dosing and scheduling regimens. The goal is to overcome or prevent drug resistance while minimizing treatment-associated toxicity.

Another challenge to the use of these new agents particularly for the BRAF inhibitors - is the unanticipated, paradoxical activation of ERK signaling in patients' cells that do not harbor the BRAF mutation [5]. Thus, in cells with wild-type $B R A F$ alleles, the current BRAF inhibitors in fact promote ERK signaling through an unintended allosteric activation of dimers of BRAF with the related kinase CRAF, leading to MEK and ERK activation. This appears to be particularly relevant in cells harboring an activated allele of NRAS, which encodes a RAS-family kinase. Indeed, approximately $20 \%$ of melanoma patients treated with the BRAF inhibitors vemurafenib or dabrafenib develop keratoacanthomas and/or squamous cell carcinomas of the skin that can harbor activating $R A S$ mutations (mostly HRAS), suggesting that the oncogenic potential of these $R A S$ mutations is realized specifically in the context of drug treatment [6]. More recent clinical evaluation of BRAF and MEK inhibitor combination treatment in melanoma suggests that this regimen reduces the incidence of these skin lesions, presumably as a result of the ability of the MEK inhibitor to suppress the activation of ERK downstream of RAS-driven BRAF:CRAF-dependent MEK activity [7].

Despite the dramatic clinical benefit associated with these new 'personalized' drug treatments for melanoma patients, the issues described above highlight some important remaining challenges, and the many published reports on this topic over the past 3 years point to the intense interest in better understanding this key pathway in melanoma. Here, I highlight two such recent studies $[8,9]$ that address the treatment of BRAF mutant melanoma, revealing new therapeutic strategies that can potentially improve outcomes for such patients. 


\section{Managing NRAS-mutant leukemia through intermittent co-treatment with BRAF and MEK inhibitors}

The first of these reports, from Paul Chapman and colleagues [8], describes a case study of a melanoma patient with a BRAF mutant tumor who was diagnosed with chronic myelomonocytic leukemia (CMML) while undergoing treatment with vemurafenib. The leukemic cells in this patient were associated with an activating NRAS G12R mutation, consistent with a potential role for vemurafenib in inducing ERK activation, similar to that seen in the RAS-mutant skin lesions observed in melanoma patients treated with BRAF inhibitors. Thus, vemurafenib treatment may have accelerated the development of a previously unsuspected RAS-mutant leukemia. The patient's melanoma was effectively managed with intermittent vemurafenib treatment, and the authors [8] observed that combining BRAF and MEK inhibitors effectively reduced the proliferation of CMML cells. Although CMMLderived DNA revealed that NRAS-mutant leukemic cells were still detectable following treatment, the patient had experienced symptomatic improvement over an 85-week treatment period, until the time of the report.

The investigators [8] also examined leukemia cell line models with or without an NRAS mutation to demonstrate that BRAF/MEK co-treatment could inhibit proliferation of NRAS mutant leukemic cells, and that, unlike BRAF inhibition alone, which promoted ERK activation, co-treatment resulted in effective suppression of ERK activation. They concluded that the use of intermittent dosing with combined MEK and BRAF inhibitors prevented the paradoxical ERK activation normally seen with sustained BRAF inhibition in cells that do not harbor an activating BRAF mutation. The authors noted that the potential anti-leukemic effects of MEK inhibitor treatment may have been partially mitigated by cotreatment with vemurafenib, which promotes ERK activation in the NRAS mutant leukemic cells. Although this study reflects the experience of a single patient, MEK inhibition appears to be effective in $R A S$-mutant myeloid malignancies, as evidenced by some early clinical studies [10], and the authors [8] suggest that intermittent treatment in this context may reduce associated drug toxicities while yielding enhanced pathway inhibition.

\section{Epidermal growth factor receptor expression in BRAF mutant melanoma}

In a second report, from Rene Bernards and colleagues [9], a novel mechanism of resistance to BRAF inhibition in melanoma was described. Several molecular mechanisms associated with acquired and intrinsic resistance to BRAF inhibition have now been reported, including activating mutations in NRAS and MEK1, amplification or alternative splicing of $B R A F$ itself, and increased expression of the COT1 kinase or various receptor tyrosine kinases [4]. Building on their previous observation of intrinsic resistance of $B R A F$ mutant colorectal cancers to epidermal growth factor receptor (EGFR) activation [11], these investigators [9] explored a potential role for EGFR in acquired resistance to BRAF inhibition in $B R A F$ mutant melanoma. Indeed, they found that posttreatment melanoma biopsies revealed evidence of increased EGFR expression.

Then, to determine how EGFR expression is regulated in melanocytes, a cell type that does not normally express EGFR, Bernards and colleagues [9] conducted an RNA interference screen using a library of short hairpin RNAs designed to target each of the more than 600 genes encoding various chromatin-associated regulatory proteins, including several histone modifying enzymes. That screen identified the SRY (sex determining region Y)-box 10 (SOX10) gene as a regulator of EGFR expression in four different tested melanoma cell lines. Moreover, SOX10 knockdown was sufficient to confer vemurafenib resistance, and this was associated with increased EGFR expression. Additional studies revealed that SOX10-mediated suppression of transforming growth factor $\beta$ receptor expression was responsible for SOX10's repressive effect on expression of EGFR as well as on other receptor tyrosine kinases that appear to contribute to vemurafenib resistance. The role of SOX10 in regulating EGFR in melanoma was further supported by the discovery of an inverse relationship between SOX10 and EGFR gene expression in a panel of BRAF mutant melanoma cell lines, as well as in a few BRAF mutant melanoma patient samples following treatment-associated resistance [9].

Interestingly, these investigators [9] had also observed that EGFR expression in BRAF mutant melanoma cells was surprisingly detrimental to their proliferative capacity, and was only tolerated in the context of BRAF inhibition. This finding suggests that, in the absence of drug, adaptive mechanisms underlying drug resistance may come at a 'fitness cost', potentially explaining clinical findings of reversible drug resistance in vemurafenib-treated melanoma patients. Thus, in patients whose cancer progresses on treatment and are consequently taken off therapy, residual tumor cells that lack the proliferation-compromising resistance mechanism (EGFR expression in this case) may have a selective growth advantage, leading to their enrichment as a dominant clone within the relapsed tumor. In such a patient, re-treatment with a BRAF inhibitor would be expected to lead to partial tumor regression. This highlights the dynamic nature of tumor cell heterogeneity in the context of treatment sensitivity and resistance, and the potentially important role of treatment scheduling in producing optimal clinical benefit.

In summary, these two recent reports [8,9] provide important new insights into some of the complexities 
associated with targeting the BRAF-MEK-ERK signaling pathway in BRAF mutant melanoma. Although they address distinct aspects of treatment-associated challenges (acquired resistance and oncogenic side effects), they both relate to the less appreciated issue of treatment scheduling. This will undoubtedly become a more prominent topic of study as both laboratory-based and clinical investigators continue to optimize strategies for combination treatments that can manage these tumors more effectively and safely.

\section{Abbreviations}

CMML: Chronic myelomonocytic leukemia; EGFR: Epidermal growth factor receptor; SOX: Sex determining region Y-box.

\section{Competing interests}

The author is an employee of Genentech, Inc. and a shareholder of Roche Pharmaceuticals.

\section{Published: 28 April 2014}

\section{References}

1. Davies H, Bignell GR, Cox C, Stephens P, Edkins S, Clegg S, Teague J, Woffendin H, Garnett MJ, Bottomley W, Davis N, Dicks E, Ewing R, Floyd Y, Gray K, Hall S, Hawes R, Hughes J, Kosmidou V, Menzies A, Mould C, Parker A, Stevens C, Watt S, Hooper S, Wilson R, Jayatilake H, Gusterson BA, Cooper C, Shipley J, et al: Mutations of the BRAF gene in human cancer. Nature 2002, 417:949-954

2. Chapman PB, Hauschild A, Robert C, Haanen JB, Ascierto P, Larkin J, Dummer R, Garbe C, Testori A, Maio M, Hogg D, Lorigan P, Lebbe C, Jouary T, Schadendorf D, Ribas A, O'Day SJ, Sosman JA, Kirkwood JM, Eggermont AM, Dreno B, Nolop K, Li J, Nelson B, Hou J, Lee RJ, Flaherty KT, McArthur GA, BRIM-3 Study Group: Improved survival with vemurafenib in melanoma with BRAF V600E mutation. N Engl J Med 2011, 364:2507-2516.

3. Flaherty KT, Robert C, Hersey P, Nathan P, Garbe C, Milhem M, Demidov LV, Hassel JC, Rutkowski P, Mohr P, Dummer R, Trefzer U, Larkin JM, Utikal J, Dreno B, Nyakas M, Middleton MR, Becker JC, Casey M, Sherman LJ, Wu FS, Ouellet D, Martin AM, Patel K, Schadendorf D, METRIC Study Group: Improved survival with MEK inhibition in BRAF-mutated melanoma. N Engl J Med 2012, 367:107-114.

4. Corcoran RB, Settleman J, Engelman JA: Potential therapeutic strategies to overcome acquired resistance to BRAF or MEK inhibitors in BRAF mutant cancers. Oncotarget 2011, 2:336-346.

5. Gibney GT, Messina JL, Fedorenko IV, Sondak VK, Smalley KS: Paradoxical oncogenesis-the long-term effects of BRAF inhibition in melanoma. Nat Rev Clin Oncol 2013, 10:390-399.

6. Sloot S, Fedorenko IV, Smalley KS, Gibney GT: Long-term effects of BRAF inhibitors in melanoma treatment: friend or foe? Expert Opin Pharmacother 2014, 15:589-592.

7. Flaherty KT, Infante JR, Daud A, Gonzalez R, Kefford RF, Sosman J, Hamid O, Schuchter L, Cebon J, Ibrahim N, Kudchadkar R, Burris HA 3rd, Falchook G, Algazi A, Lewis K, Long GV, Puzanov I, Lebowitz P, Singh A, Little S, Sun P, Allred A, Ouellet D, Kim KB, Patel K, Weber J: Combined BRAF and MEK inhibition in melanoma with BRAF V600 mutations. N Engl J Med 2012, 367:1694-1703.

8. Abdel-Wahab O, Klimek VM, Gaskell A, Viale A, Cheng D, Kim E, Rampal R, Bluth M, Harding JJ, Callahan MK, Merghoob T, Berger MF, Solit DB, Rosen $N$, Levine RL, Chapman PB: Efficacy of intermittent combined RAF and MEK inhibition in a patient with concurrent BRAF and NRAS mutant malignancies. Cancer Discov 2014.

9. Sun C, Wang L, Huang S, Heynen GJ, Prahallad A, Robert C, Haanen J, Blank C, Wesseling J, Willems SM, Zecchin D, Hobor S, Bajpe PK, Lieftink C, Mateus C, Vagner S, Grernrum W, Hofland I, Schlicker A, Wessels LF, Beijersbergen RL, Bardelli A, Di Nicolantonio F, Eggermont AM, Bernards R: Reversible and adaptive resistance to BRAF(V600E) inhibition in melanoma. Nature 2014.

10. Jain N, Curran E, lyengar NM, Diaz-Flores E, Kunnavakkam R, Popplewell L, Kirschbaum MH, Karrison T, Erba HP, Green M, Poire X, Koval G, Shannon K,
Reddy PL, Joseph L, Atallah EL, Dy P, Thomas SP, Smith SE, Doyle LA, Stadler WM, Larson RA, Stock W, Odenike O: Phase II study of the oral MEK inhibitor selumetinib in advanced acute myelogenous leukemia: a University of Chicago phase II consortium trial. Clin Cancer Res 2014, 20:490-498.

11. Prahallad A, Sun C, Huang S, Di Nicolantonio F, Salazar R, Zecchin D, Beijersbergen RL, Bardelli A, Bernards R: Unresponsiveness of colon cancer to BRAF(V600E) inhibition through feedback activation of EGFR. Nature 2012, 483:100-103.

doi:10.1186/gm547

Cite this article as: Settleman: Optimizing the treatment of BRAF mutant melanoma. Genome Medicine 2014 6:30 